\title{
MRI Brain Image Segmentation using Modified Fuzzy Logic Clustering (MFLC)
}

\author{
Reda Shbib \\ Department of Computer Science, \\ International University of Beirut, Mazraa, Beirut,
} Lebanon

\author{
Hussein Trabulsi, Hala Sabagh \\ Department of Management information System, \\ Lebanese University, Beirut, \\ Lebanon
}

\begin{abstract}
Brain Tumor is a development of abnormal cells in the tissues of the brain and could be life menacing. It has critical impact on the person's emotions, personality and quality of life. However, not all brain tumors are cancers. An early diagnosis increases the chance of a patient to survive. Various techniques were developed for this purpose. One of the common techniques for brain tumor recognition is MRI (Magnetic Resonance Imaging). The segmentation of MRI images is an important task to analyze brain changes. It aims to differentiate between normal and abnormal tissues in order to provide the corresponding treatments. However, ensuring an efficient and accurate segmentation is a challenging task due to the different tissues in the brain that varies in shape and size. In this paper, we propose a new technique called Modified Fuzzy Logic Clustering (MFLC) for brain tumor detection in MRI images. Our experimental results and analyses indicate that our proposed technique is superior in detecting brain tumors in comparison to $\mathrm{K}$-means.
\end{abstract}

\section{INTRODUCTION}

The causes of brain tumors are unexplored [1], however there are risk factors that increase the chance of developing tumors. Exposure to radiations on the head may raise a person's risk; For example, children with ringworm of the scalp who are most of the time treated with radiation, people who have neurofibromatosis or Li-Fraumeni syndrome [2] and many more cases have high chance to be threatened by the tumors as they get older. People between the ages of 65 and 79 are the most likely to be diagnosed with a brain tumor. The age factor depends on the cell type, tumor size and location. However, those cases represent a fraction of the approximately 28,000 new primary brain tumors diagnosed each year in the United States [3].

A primary brain tumor is one that commences in the brain, but not all primary brain tumors are cancerous; a tumor can be benign or malignant. Benign tumor is not aggressive. It does not expand beyond its borders to surrounding tissues, although it can be serious and even life threatening when it presses on sensitive areas of the brain. Malignant tumors are cancerous invasive cells, which do intrude into the surrounding healthy tissue and spread to other part of brain or spine. Tumor cells grow very faster and graver than benign tumor. Unlike benign tumors, they have no clear borders. Their boundaries are irregular and hard to be marked off due to the invasion to normal tissues. Therefore, the automated detection and segmentation of brain region and abnormalities are very important in the context of accurate measurement and time. Segmentation of Gray Matter (GM) and White Matter (WM) of the brain is also very effective on several diseases [4]. However, manual segmentation of WM and GM is very timeconsuming as well as tiresome. In this work, we propose a clustering algorithm for WM and GM segmentation to provide low error rate and fast accurate results.

This paper is organized in the following main sections. Section 2 provides an overview of the brain tissues and MRI technique. In section 3 , we discuss the current different brain tumor segmentation techniques. Section 4 describes the steps of our proposed method. Experimentation results and evaluation are presented in Section 5, and Section 6 concludes our work.

\section{PRELIMINARIES}

\section{A. Brain tissues}

Generally, Brain tissue has been classified into two main classes. First Class is the Gray matter and the second is the white matter. For long Time, White matter (WM) has been a main and significant area of study in computer vison area especially in the medical and imaging field of MRI. It is considered as the highway information path of the brain that speed up the linkage between the different portions of the brain and the other body parts. Gray matter (GM) is dark "in colour". It involves unmyelinated neurons, what so called "'glial cells" which are the controller of most of the brain activities. White matter surrounds the gray matter [5]. It is made up of neurons, which is called myelinated that make an interconnection between gray matter in addition to the other body parts.

\section{B. Brain tissues}

Magnetic resonance imaging (MRI) is usually used to identify brain tumors. It creates an image of the brain, and processes it using segmentation. MRI helps in evaluating brain's size and its constituent volumes (grey matter, white matter, cerebrospinal fluid (CSF)). Three primary types of medical imaging planes are utilized in neuroimaging: 
- Axial plane (Figure 1.1-a): Transverse planes; images represent "slices" of the body.

- Sagittal plane (Figure 1MRI - Medical imaging Planes): Images taken perpendicular to the axial plane, which bisects the left from the right sides (lateral figure).

- Coronal plane (Figure 1 c): Images taken perpendicular to the sagittal plane, which bisects the front from the back. (Frontal view).

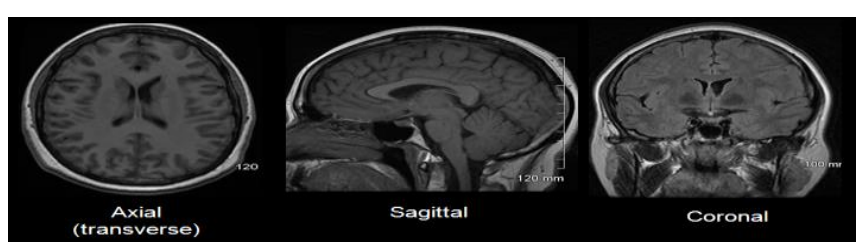

Figure 1MRI - Medical imaging Planes

For the early detection of brain tumor, different imaging techniques were proposed: Positron Emission Tomography (PET), Magnetic Resonance Imaging (MRI), and Computed Tomography (CT). MRI is proven to be most efficient for the brain tumor detection and classification as compared to other imaging techniques. An MRI device make use of a magnetic and radio wave in order to obtain clear and fully comprehensive details of the images in the brain. It does not release any damaging radiation, which can affect the body

\section{RELATED WORK.}

Manual segmentation has long been used to detect brain tumors. It refers to the process where an expert physician segments and labels an image by hand. This is done in a "sliceby-slice" way to correctly reconstruct $3 \mathrm{D}$ object imagery from different adjacent 2D images. However, this task is time consuming thus inadequate for large studies or daily clinical use $[5,6,29]$. To overcome this, accurate automation of segmentation (fully or partially) is needed.

\section{A Semi-Automatic}

Most of the current research is targeted at semi-automatic segmentation of brain tumors with the intention of having the least human interaction possible. In semi-automatic segmentation, human intervention is needed to initialize the method that will be used and to check how well the segmentation have been accurately obtained and assist doctors in qualitative diagnosis. Sometimes, it is required to manually correct the results' segmentations.

Several methods have been offered for brain tumours classification in the magnetic resonance pictures, utmost especially, fuzzy - machine (SVM), artificial neural network (ANN) means support vector machine techniques. These techniques are commonly used for region-based segmentation to obtain significant data from the medical images [7]. Below we present a summary of the current and noticeable studies covered in this literature.

Damodharan [8] have implemented a technique using a (NN) for detection of the tumour. In his proposed technique, the rate is formed distinctly for the segmentation of WM, GM, and tumour region of interest. The author states an accurate level of $83 \%(\mathrm{NN})$ based on some classifier.

Salem et al [9] have implemented a method to automate the classification of tumour from MR images. SBM (single brain metastasis) has been used as classifier using a SVM based classifier. To increase its accuracy level of the classifier, some features has been extracted by applying the fast Fourier transform (FFT). The reduction of features has been achieved by making the minimal- Redundancy-Maximal-Relevance method. This method has achieved an accuracy level of $87 \%$. Brain tumour extraction techniques necessitates the partitioning of the brain images to two separate areas [10]. The first one comprises the cells which constitutes the cells of the brain and the other part comprises the brain cells which considered as normal [11].

In his work, Zanaty [12] has presented an approach for the segmentation of brain tumour based on a mixture kind of methods: merging FCM, seed area growing, and Jaccard resemblance coefficient technique, which has been used for measurement of the segmented GM and WM tissues from MRI images. The proposed algorithm achieves a typical segmentation $90 \%$ based on the level of image noise of 3\% and $9 \%$, correspondingly. Kong [13] has implemented an automated segmentation process of brain tissues from MRI images by make use of selection method. In addition, Demirhan [14] has offered a new technique for the segmentation of tissue by making use of wavelets NN.

Torheim [15] and Guo. [16] have proposed a method, which make use of texture features, wavelet transform, and the support vector machine in order to obtain an efficient classification of contrast improved MR images, to deal with the nonlinearity of real information and to point out for diverse image protocols successfully. In addition, Torheim [15] stated that their suggested system provides much better calculations and better-quality scientific factors, such as the volume and the shape of the tumor, and tumor phase comparing through first-order arithmetic features.

Kumar [17] presented a method for brain tumor classification and segmentation. It depends on the radial basis function (RBF) kernel that mainly rely on the Support Vector machine and states a matching index of $96.1 \%$, overlapping ration of $94 \%$, the accurate classification level was around $94 \%$ with ratio errors of $7 \%$.

Sharma [18] has introduced a good and efficient method, which states a good level of accuracy. This technique make use of texture-primitive features through (ANN) for segmenting and classifying.

Cui [19] introduced a local based fuzzy clustering with spatial data to construct an objective of medical image segmentation and bias field in order to estimate the MRI images. In his technique, the authors make use of "Jaccard" matching as an index to measure how well the accuracy rate. The author attain $83 \%$ of an accuracy white matter, gray matter segmentations. A1 Wang [20] have introduced a segmentation technique, which is based on active silhouette technique to handle the problematic issues of the inhomogeneity. Deepa [21] has presented a method using a learning machine in order to classify the brain tumor from MRI. The proposed method has reached a level of accuracy 
around $92 \%$, in addition to sensitivity and specificity level of $91 \%, 96 \%$ respectively.

The real communication between the device and the operator can be done through the output and input computerized machine in which can be controlled by the graphical used user interface (GUI). The operator at that time will investigates the graphical information shown on the device and responds consequently, giving some feedback for the computation. The operator communication in brain tumour segmentation have been assembled by Foo [23] into three key classes: the initialization part, then the involvement or feedback reply, and finally the assessment. Most of approaches involve of one or further kinds of involvement

Meanwhile the semiautomatic approaches make use of diverse policies to make a combination between devices and operator's expertise. The consequence of such approaches hangs on the approach as much as on calculation. Such policies might comprise connecting the operator in the initialization of segmentation, keeping the operator in control during the entire procedure, or adding intellectual behavior to raise the intellection of collaboration. Even though it is factual that by applying such techniques strategies, effective detection of tumour semiautomatic approaches would be attained, semi-automatic segmentation comparable to manual segmentation is exposed to variants both between skilled operator and within the same operator.

\section{B Unsupervised Methods}

Many of the unsupervised systems are clustering based and not reliant on training-data. The mainly frequently adopted procedures for clustering are $\mathrm{K}$ and Hard C-means or Fuzzy C-means. K-means technique [24] yields to inaccurate segmentation. However fuzzy segmentation process is much smoother and can be changed into solid segmentation by letting the pixels have membership of the cluster that has the maximum weight of the coefficient's membership.

Using clustering technique, the mainly purpose is to build some decision boundaries which are built on training data that are unlabelled. Generally, Clustering can be defined based on the procedure of obtaining and finding natural group of clusters based on multi-dimensional feature space. It is somehow problematic, since clusters of diverse forms and dimensions can arise in multidimensional feature space. An amount of efficient descriptions of clusters has been proposed in the literature. Arrangements within a cluster are more alike to each other than arrangements fitting into different clusters. Yet, the key restrictions of fuzzy clustering process are: (a) sensitivity to preliminary partition matrix (b) discontinuing criterion (c) result might come to be held at local minima. Therefore, clustering methods could not end with best result and there is no best clustering technique for a precise use. An amount of dissimilar processes is obligatory to be attempted to catch the optimal technique one.

There are many Brain tumour types and the methods mentioned here either focus on the most common tumour types, i.e. glioblastoma, or require specific training database to deal with a specific tumour type. Only few researchers, tried to train a developed algorithm on one tumour type and test it on another. However, the results were not satisfying. Many methods were developed to get feature extraction and others were developed to tackle only classification. However, the extraction of Feature in addition to the reduction of feature vectors for operative segmentation of WM, GM, and diseased tumor area as well as the examination on joint method might not be shown in all the available works. Furthermore, only rare features have been extracted and consequently very little level of accuracy by means of tumour detection has been gotten.

\section{PROPOSED WORK}

Segmentation is a significant phase in the medical processing. The purpose of segmentation is to segment an image into important areas with respect to a particular application. In other words, it is the task of splitting a digital image into one or more regions of interest. This process is based on measurements taken from the image and might be grey level, colour, texture, depth or motion.

\section{A Overview}

During the process of segmentation, an accurate detection of abnormalities in the brain from MRI is needed. This task is very important because the presence of artefacts in MRI degrades the image and the diagnostic quality. Achieving an accurate detection of GM and WM boundaries is an important task. The technique is divided into two stages, pre-processing (consists of image enhancement, noise and skull stripping) and post-processing stage (involves GM/WM segmentation and clustering).

Image pre-processing and feature extraction techniques are mandatory for any image-based applications. The accuracy and convergence rate of such techniques must be significantly high in order to ensure the success of the subsequent steps. An effective method for binarization, artefact and skull removal is used because artefacts and skull may hamper the automated detection. Figure 3, shows the final state of an image after skull-stripped. Moreover, Acquired image consists of speckle noise and is usually of low contrast. To overcome speckle noise, and low contrast, pre-processing of image is required. Below we detail the main steps of the proposed segmentation method.

\section{B Segmentation: steps and challenges}

\section{$1 \quad$ Noise removal}

Noise can be described as the random change of the ideal pixel value in the acquisition phase. That is to say, now when we can first perceive a measured pixel value, it has been distorted from its ideal value by a random component. Any Imaging system experiences noise to some extent. We can model the effect of noise by:

$$
G(x, y)=f(x, y)+\eta(x, y)
$$

Where $\mathrm{G}(\mathrm{x}, \mathrm{y})$ is the image we observe, $\mathrm{f}(\mathrm{x}, \mathrm{y})$ represents the unknown ideal (undistorted) image, and $\eta(x, y)$ is the noise component. Because noise is inherently a random element in the imaging process, we are unable to reconstruct the ideal pixel values $\mathrm{f}$ from the observed values $\mathrm{g}$. It is, however, 
usually possible to predict some global effects of noise, if we know something of the statistical behaviour of the noise $\eta$. In other words, if we have some knowledge on the statistical distribution of the stochastic variable $\eta$.

The Big problem which occurs in MRI images is the very low contrast, this is because the effect of noises and some additional magnetic field. For such reason, and in order to improve the result of segmentation, it was required to apply a smoothing filter before going into the segmentation stage.

Additional problematic part that has been faced during this stage of was the existence of skull. Yet skull is a vital portion in the human, however it is not required for analysing using MRI. Therefore, it should be removed. To deal with this problem, morphological operation based on some strutting element has been used.

\section{$2 \quad$ Skull stripping}

Skull stripping is a significant procedure in medical analysis. It is essential for the effectiveness of the inspection of brain [25-28]. Skull stripping is the procedure of removing all tissues, which are considered as non-brain like fat, membrane, and head in the images. There exist numerous methods for stripping. Some of the widespread methods are automated stripping utilizing image silhouette. Some of them are based on morphological operation, histogram analysis or a thresholding algorithm. Figure 2 shows the phases of the stripping procedure. This paper makes use of the skull stripping method, which mainly rely on a thresholding process to take out skull tissues

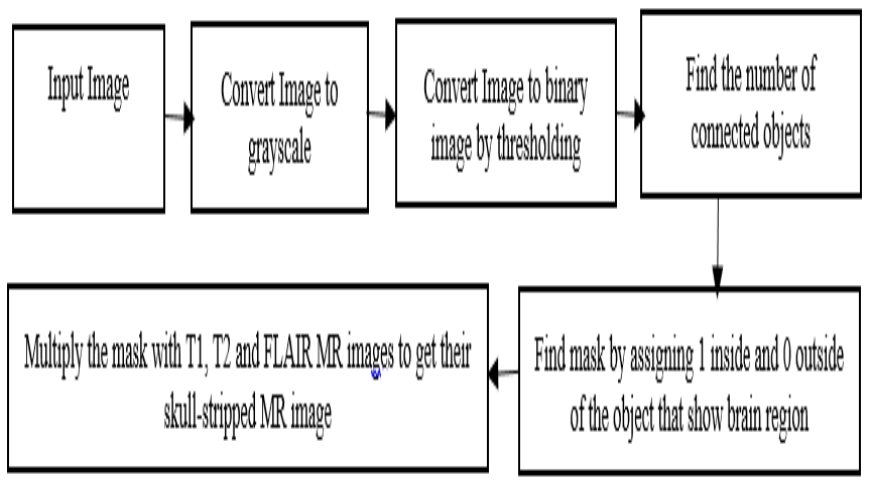

Figure 2 Steps for skull stripping

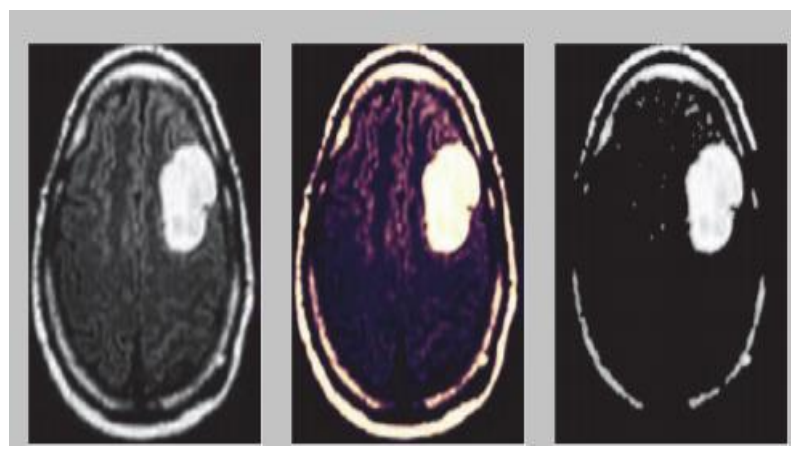

Figure 3 : (a) Original image

(b) Enhanced image (c) Image after Skullstripped

\section{Clustering}

Clustering is defined as the process of identifying groups of similar image primitive. An image can be grouped based on keyword (metadata) or its content (description). The "keyword" is form of font which describes about the image keyword of an image refers to its different features. The "content" refers to shapes, textures or any other information that can be inherited from the image itself.

In this paper, both k- and Fuzzy c-Means techniques have been combined together to obtain our special segmentation algorithms. In addition to clustering techniques based on pixel intensity weight, and some arithmetical based on the values of the pixels.

\section{Quick overview}

a) K-Means

K-Means follows a numerical, unsupervised, nondeterministic and iterative method for partitioning images into $\mathrm{k}$ clusters. Selection of $\mathrm{k}$ can be done manually, heuristically and randomly. At first, the pixels are clustered based on their color, intensity, texture, and location or a weighted combination of these factors where the clustering process is accomplished. The quality of the solution depends on the initial set of clusters and the value of $\mathrm{k}$. A good clustering will produce high quality clusters with high intra-class similarity and low inter-class similarity. The main challenge with the kmeans algorithm is the selection of initial centroid. If the initial centroid selection is not proper, it will affect the result of the clustering.

\section{b) Fuzzy C- Means}

C-Fuzzy clusters are created depending on the data and the application. Different types of similarity measures may be used to identify clusters. This algorithm is based on the minimization of following objective function value:

$$
J=\sum_{i=1}^{N} \sum_{j=1}^{C} \mu_{i j}^{m}\left|x_{i}-c_{j}\right|^{2}
$$

Where $\mathrm{J}$ is the objective function, $\mathrm{N}$ is the number of pixels in the image, $\mathrm{C}$ is the number of clusters, $\mu$ is the membership table -- a table of $\mathrm{NxC}$ entries which contains the membership values of each data point and each cluster, $\mathrm{m}$ is a fuzziness factor (a value larger than 1), xi is the ith pixel in $\mathrm{N}$, cj is jth cluster in $\mathrm{C}$ and $|\mathrm{xi}-\mathrm{cj}|$ is the Euclidean distance between $\mathrm{xi}$ and ci.

\section{Our proposed clustering algorithm}

1. Browse the file path; select the image to be processed (JPEG format) from database of MRI images.

2. Check if the image is RGB then convert it to gray image.

3. Convert the image to double type to increase the range of pixel values.

4. For Fuzzy C-means (FCM), predefine the number of iterations and number of clusters.

5. Get the size of the image.

6. Convert input image matrix to a vector. 
7. Randomly select the k cluster centre.

8. Calculate the fuzzy centre (vector) using the formula in equation (2).

9. Calculate the fuzzy membership function using distance formula in equation (3).

10. Repeat steps 8 and 9 until a minimum value is achieved of equation (4).

11. Terminate if stopping condition in equation (5) is true otherwise return to step 8 .

12. Combine the membership grades and class values of clusters; map and reshape the respective pixels to form the final clustered image.

Fuzzy C-means is the clustering algorithm, which allows one piece of data to be a member of more than one clusters. It is based on reducing the following function:

$$
Y_{m}=\sum_{i=1}^{N} \sum_{j=1}^{c} M_{i j}^{m}\left\|x_{i}-c_{i j}\right\|
$$

Where,

$\mathrm{m}$ - Real number greater than 1 ,

Mij- membership's degree of $\mathrm{x}$; within the cluster $\mathrm{j}$,

$\mathrm{Xi}$ - data measured in d-dimensional,

$\mathrm{Rj}$ - d-dimension centre of the cluster (vector),

$$
d(A, B)=\frac{1}{n} \sum_{1}^{n}\left|\mu_{A}\left(x_{i}\right)-\mu_{B}\left(x_{i}\right)\right|
$$

The update of Fuzzy membership $\mathbf{M}_{\mathrm{ij}}$ and the cluster centres $\mathrm{R}$ are given by

$$
R_{i}=\sum_{1}^{N} x_{i} M_{i j}^{m} / \sum_{1}^{N} M_{i j}^{m}
$$

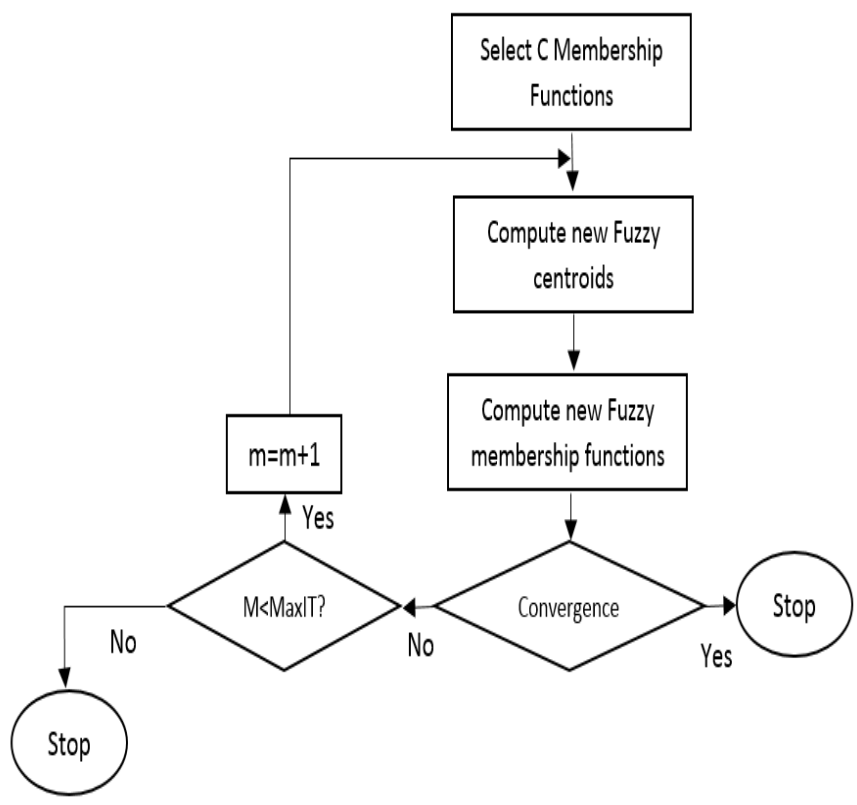

Figure 4 Clustering algorithm in our work

\section{Smoothing and Filtering}

Image restoration is an important step in the reconstruction process. The final appearance and the quality of the reconstructed image depend greatly on the restoration method used. In addition, the purpose of image restoration is to reduce the degradations that are caused during acquisition of US scanning. In this system for proper orientation, level set function is used. By the use of plan curve motion and curve smoothers, shrinks are eventually disappeared.
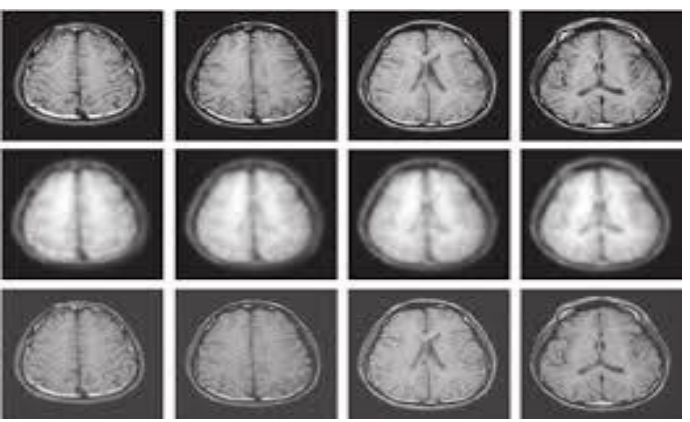

MRI Smoothing

To obtain optimal resolution in both spatial and frequency domains often done to reduce the effect of pixel noise in images, Gabor filters with different frequencies and with orientations in different directions have been used to localize the band pass filter for the local spatial frequency distribution. The standard deviation of the Gaussian function can be varied to adjust degree of smoothening.

The median filter is a nonlinear digital filtering technique, often used to remove noise from the image. It is very widely used in image processing because, under certain conditions, it preserves edges while removing noise. The main idea of the median filter is to run through all pixels value one by one which are sorted into numerical order, and then replacing each one with the median of neighbouring entries. Median filtering is similar to using a standard mean filter, in that every pixel would be the "average" of the neighbourhood values.
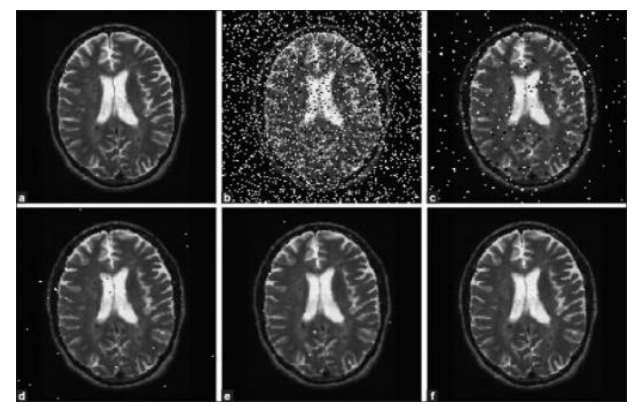

Features Extraction and Classification

\section{E-1 Statistical features}

The statistical features that have been used for extraction in our proposed work are mean, variance, standard deviation, 
The mean: It is computed by adding all the values the pixels of an image and then dividing by the overall number of pixels.

$$
M=\left(\frac{1}{m \times n}\right) \sum_{x=0}^{m-1} \sum_{y=0}^{n-1} f(x, y)
$$

Standard Deviation (SD): It is the additional essential moment defining probability spreading of a perceived population and be able to help as a measurement of inhomogeneity. A greater value specifies an improved level of intensity and great contrast of edges.

$$
s=\sqrt{\frac{\sum_{i=1}^{N}\left(x_{i}-\bar{x}\right)^{2}}{N-1}}
$$

Entropy: It is intended to describe the arbitrariness of the texture in an image.

$$
\begin{aligned}
f(x)=a_{0}+\sum_{n=1}^{\infty}\left(a_{n} \cos \frac{n \pi x}{L}+b_{n} \sin \frac{n \pi x}{L}\right)+f(x) \\
=a_{0}+\sum_{n=1}^{\infty}\left(a_{n} \cos \frac{n \pi x}{L}+b_{n} \sin \frac{n \pi x}{L}\right)
\end{aligned}
$$

- Contrast: It is the measurement of the pixel's intensity its neighbour within an image,

$$
C_{o n}=\sum_{x=0}^{m-1} \sum_{y=0}^{n-1}(x)
$$

- Structured Similarity Index (SSIM). It is a perception factor that indicates that the degeneracy in the quality of the image might be produced by the compression of data or damages in data while transmission. It is well-defined as

$$
\begin{aligned}
& \operatorname{SSIM} \\
& =\left(\frac{\sigma_{x y}}{\sigma_{x} \sigma_{y}}\right)\left(\frac{2 \overline{x y}}{\left(\bar{x}^{2}\right)+\left(\bar{y}^{2}\right)+C_{1}}\right)\left(\frac{2 \sigma_{x} \sigma_{y}}{\left(\sigma_{x}\right)^{2}+\left(\sigma_{y}\right)^{2}+C_{2}}\right)
\end{aligned}
$$

- Mean Square Errors. The precision of a technique is the degree of depth of how near the estimate is from the factual values. The predictive effectiveness of the proposed method is assessed by the below error measurements: Mean Absolute Error (MAE), the mean square error (MSE) and mean relative error (MRE).

$$
M A E=\frac{1}{n} \sum_{t \in n}\left|\mu_{t}^{E}-y_{i}\right|
$$

Where $f_{i}$ signifies the likelihood by the method and $y_{i}$ signifies the factual rate

$$
\begin{gathered}
\text { MSE }=\frac{1}{n} \quad \sum_{t \in n}\left|\mu_{t}^{E}-y_{i}\right|^{2} \\
M R E=\frac{1}{n} \sum_{t \in n}\left|\frac{\mu_{t}^{E}-y_{i}}{y_{i}}\right| \times 100 \%
\end{gathered}
$$

Where $\mu_{t}^{E} \quad$ signifies the likely values and $y_{i}$ is the factual cost

\section{E-2 Selected Feature Extraction}

Designated features are categorized into (Shape), Entropy (E), and General-Feature (GP) categories. We have used a combination between of these features are assessed. By making use of cross validation, the ratio of the error ratios is calculated in terms of MAE, MSE, and MAE.

\begin{tabular}{llll}
\hline $\begin{array}{l}\text { Selected } \\
\text { Features }\end{array}$ & MAE & MSE & MRE \\
\hline S & 3.13 & 9.45 & $23.12 \%$ \\
E & 4.8 & 34.11 & $27.09 \%$ \\
GP & 2.67 & 10.17 & $18.35 \%$ \\
S+E & 2.88 & 8.36 & $16.56 \%$ \\
S+GP & 3.12 & $\mathbf{8 . 4 4}$ & $\mathbf{2 2 . 8 7 \%}$ \\
E+GP & 2.14 & 9.67 & $17.8 \%$ \\
S+E+GP & 2.13 & 8.765 & $16.12 \%$ \\
\hline
\end{tabular}

The performance of the used feature is much better than features used by other works, and it is distinguished, that there is reduction in performance when any feature is lacking from the whole set of features

\section{E3-Support Vector Machine}

The Support Vector Machine [25] process relies on the learning of supervised learning method and is functional from 1-class classification to many-class classification $[1,26]$. The standard purpose of the Support Vector Machine system is intended to convert a nonlinear separating objective to a linear transformation by making use of a kernel function. In this paper, we used of the Gaussian function in order to make the transformation. Using such function, nonlinear samples would be converted into a high-dimensional future space where the separation of nonlinear samples or data would turn out possible the classification useful [27]. The Support Vector Machine algorithm defined as follows:

$$
f(y)=Z^{T} \emptyset(y)+b
$$

Where $Z$ and $T$ represents hyperplane factors and $\phi(y)$ is the function that has been used for mapping the $y$ to a higher dimensional space. Equation (13) illustrates the Gaussian function of nonlinear Support Vector [28] that has been used for the best clarification of classification and generality and its function is represented in equation (14). 


$$
\begin{gathered}
k\left(y_{i}, y_{j}\right)=\exp \left[-\gamma\left\|y_{i}-y_{j}\right\|^{2}\right], \\
k\left(y_{i}, y_{j}\right)=\sum_{i=1}^{N} \sum_{X_{i} \in M_{j}}\left(\exp \left[-\gamma\left\|y_{i}-y_{j}\right\|^{2}\right]\right)
\end{gathered}
$$

Table 2 Level Feature evaluation the discriminate features for the first-order numerical

\begin{tabular}{lcc}
\hline Images & Mean & Standard deviatior \\
\hline Image 1 & 8.66 & 43.99 \\
Image 2 & 11.81 & 49.11 \\
Image 3 & 39.40 & 75.59 \\
Image 4 & 6.83 & 39.45 \\
Image 5 & 11.90 & 38.81 \\
Image 6 & 5.33 & 28.95 \\
\hline
\end{tabular}

\section{EVALUATION AND RESULTS}

Our proposed work aims to achieve accurate extractions of WM and GM, so image must contain only white and gravy matter. All the above steps describe the procedure of the whole work. Our proposed method produces very good outputs for each type of GM extraction. We used MATLAB, for data analysis, development of algorithms and creation of models and applications. The language, tools and built-in math functions in MATLAB enable us to explore multiple approaches and reach a solution faster than with spreadsheets or traditional programming languages, such as $\mathrm{C} / \mathrm{C}++$ or Java.

\subsection{Tumor Image Database}

In this paper, 500 Ultrasound Scan (US) Tumour images are collected from different hospitals of different patients and stored in a database. We chose from the database the images subjected to tumour detection. For the simulation, we have used a benchmark database, which comprised trial images.

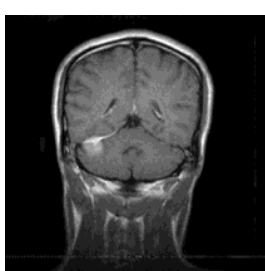

(a1)

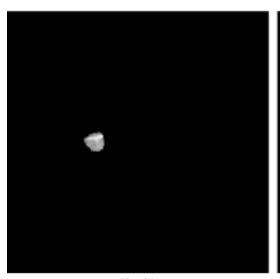

(b1)

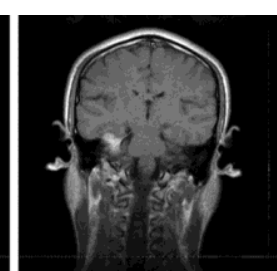

(a2)

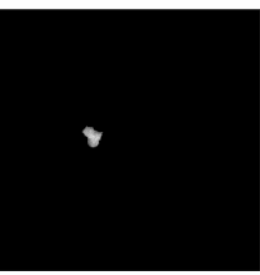

(b2)

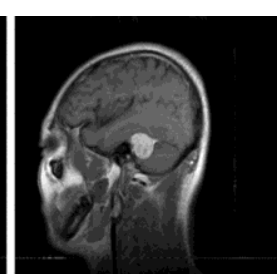

(a3)

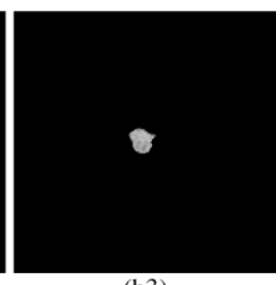

(b3)
Figure 5:Comparison of segmentation results on a $\mathrm{K}$ means (the proposed MLFC

The brain tumor images are in non-medical format (.jpg, .png, .bmp). This dataset comprised a selection of portion thicknesses, with some noisy levels, and non-uniform intensity level. The medical images utilised for our examination have $\mathrm{mm}$ slice of level of thickness, $4 \%$ noisy, and $25 \%$ of non-uniform intensity level. In this dataset, 15 out of 40 images comprised are image tumour- diseased.

\subsection{Measures of efficiency}

In this section, the methods proposed has been compared with some ground truth image of brain image. To assess the performance of the proposed algorithm, we measured efficiencies based on four parameters described below.

- TP: True Positive; Tumor Present and detected

- TN: True Negative; Tumor not present and not detected

- FP: False Positive; Tumor not present and detected

- FN: False Negative; Tumor present and not detected

- $\quad$ Efficiency $=(\mathrm{TN}+\mathrm{TP}) /(\mathrm{TP}+\mathrm{TN}+\mathrm{FP}+\mathrm{FN})$

Table 3 Quality parameters

\begin{tabular}{|c|c|c|c|}
\hline \multirow{2}{*}{$\begin{array}{c}\text { Expected } \\
\text { results }\end{array}$} & \multicolumn{2}{|c|}{ Ground truth } & \multirow{2}{*}{ Total } \\
\cline { 2 - 3 } & Positivity & Positivity & \\
\hline Positivity & TP & FN & TP+FN \\
\hline Negativity & FN & TP & FN+TP \\
\hline Total & TP+FN & FN+TP & TP+FN+FN+TP \\
\hline
\end{tabular}

\section{RESULTS}

Table 5 provides information of the diverse performance factors like (MSE), (SSIM), and dice score. A minor rate of MSE and a greater rate of PSNR designate well signal-to-noise ratio within the extracted image.

The dice Coefficient identifies the overlapping of the automated segmentation and manual segmentation for a specified dataset.

Table5 Statistical features

\begin{tabular}{lccc}
\hline Images & SSIM & MSE & $\begin{array}{c}\text { Dice } \\
\text { score }\end{array}$ \\
\hline Image 1 & 0.9876 & 9.314 & 0.91 \\
Image 2 & 0.8765 & 34.805 & 0.86 \\
Image 3 & 0.5675 & 10.186 & 0.578 \\
Image 4 & 0.3245 & 8.725 & 0.712 \\
Image 5 & 0.6754 & $\mathbf{8 . 5 3 8}$ & 0.823 \\
Image 6 & 0.8879 & 9.794 & 0.879 \\
Image 7 & 0.7123 & 8.174 & 0.73 \\
\hline
\end{tabular}

Error! Reference source not found.shows the results from applying the K means MLFC for the segmentation of noisy brain images. Error! Reference source not found.shows that we still have lots of noise when segmenting the MRI Image.

Error! Reference source not found.has shown a better segmentation than the $\mathrm{K}$ means when compared to our proposed algorithm, as shown most of noise has been removed. 
Error! Reference source not found.shows the results of applying MLFC algorithm to segment an MRI brain image. Strong homogeneities are apparent in the image. The MLFC algorithm segments the image into three classes corresponding to background, GM and WM.
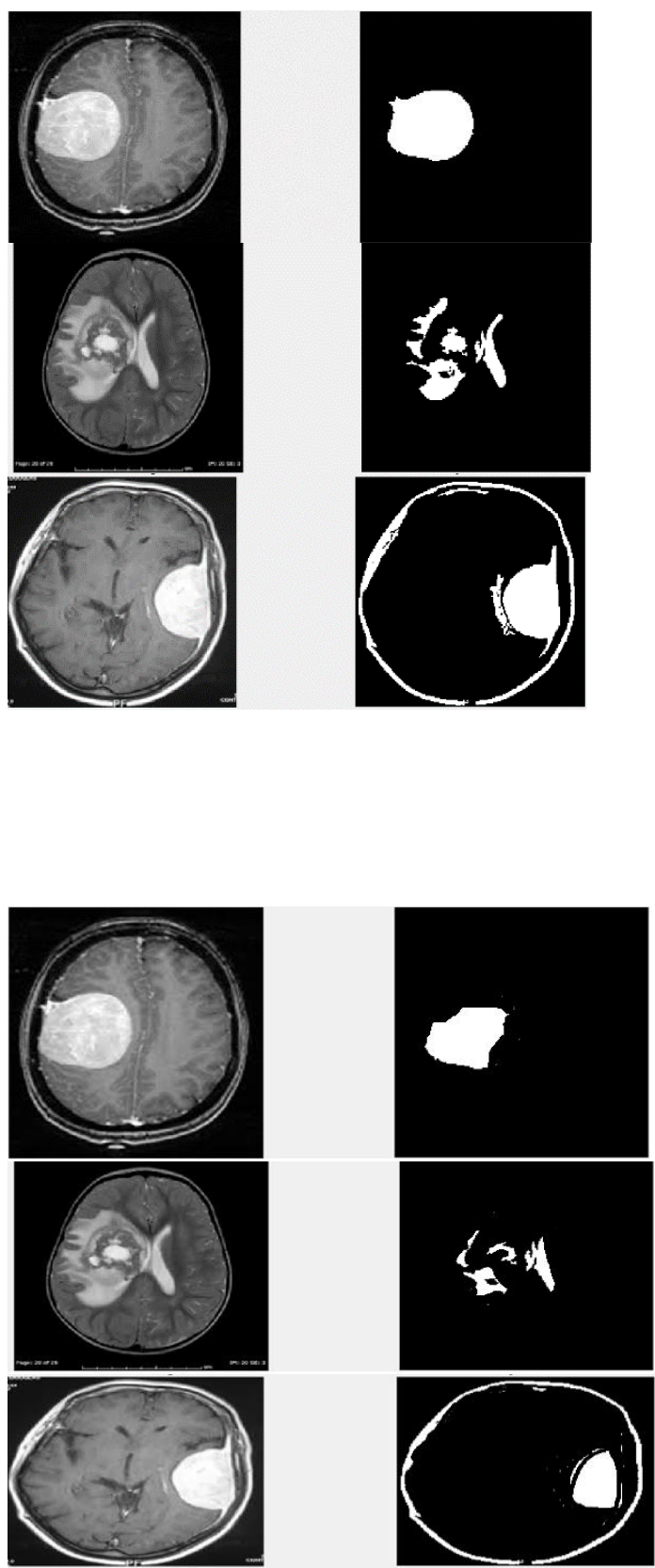

Figure 6: $\mathrm{K}$ means algorithm for tumor detection

\section{VALIDATION}

Validation presentation displays the relation between the result and the target. Sensitivity is the percentage of true positive value, which has been properly assessed by the investigational examination. Specificity is the true negative value measured by the experimental test. Correctness displays that analytical test is nearby the accurate value. The proposed technique records that sensitivity has reached an

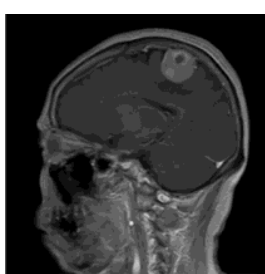

(a1)

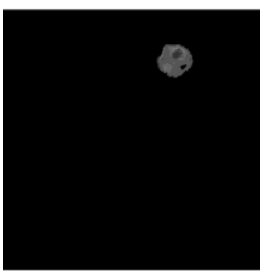

(b1)

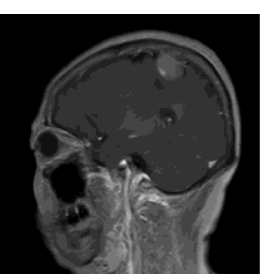

(a2)

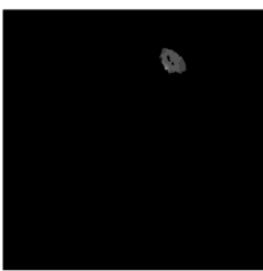

(b2)

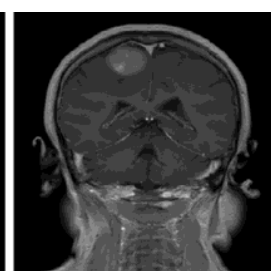

(a3)

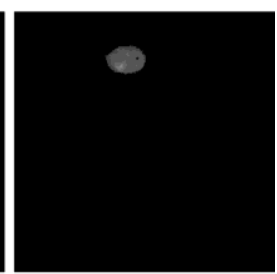

(b3) accurate results for around $95.5 \%$. In addition, the specificity has recorded a value of $97.2 \%$ and the accuracy $97.2 \%$ as shown in Table 5. Table 5 displays the evaluation compared to the related works.

Table 5 related work comparisons with our work

\begin{tabular}{ll}
\hline Related works & Accuracy \\
\hline Damodharan & $83 \%$ \\
Alfonse, et al & $87.5 \%$ \\
Demirhan & $92.2 \%$ \\
Zanaty, et al. & $92.4 \%$ \\
Proposed Method & $97.2 \%$ \\
\hline
\end{tabular}

\section{CONCLUSION}

In this paper, we used pre-processing to improve the signalto-noise ratio and to eliminate the effect of unwanted noise. We used a skull-stripping algorithm based on threshold technique to improve the skull stripping performance. Furthermore, we used MFLC to segment the images, support and to classify the tumor stage by analyzing feature vectors and area of the tumor. In this study, we investigated texturebased features with a commonly recognized classifier for the classification of brain tumor. From the experimental results performed on the different images, it is clear that the analysis for the brain tumor detection is fast and accurate when compared to the manual detection performed by radiologists or clinical experts.

The various performance factors also indicate that the proposed algorithm provides better result by improving certain parameters such as mean and accuracy. Our experimental results show that the proposed approach can help in the accurate and timely detection of brain tumour along with the identification of its exact location. Thus, the proposed approach is significant for brain tumour detection from MR images.

\section{REFERENCES}

[1] Joshi, Deepika, and Rohit Goyal. "Review of Tumor Detection in Brain MRI Images." (2017).

[2] Abbasi, Solmaz, and Farshad Tajeripour. "Detection of brain tumor in 3D MRI images using local binary patterns and histogram orientation gradient." Neurocomputing 219 (2017): 526-535.

[3] M. Rakesh, T. Ravi - Image Segmentation and Detection of Tumour Objects in MR Brain Images Using FUZZY C-MEANS (FCM) 
Algorithm" International Journal of Engineering Research and Applications 2012,6,(5), pp.2088-2094

[4] Litjens G, Kooi T, Bejnordi BE, Setio AA, Ciompi F, Ghafoorian M, van der Laak JA, van Ginneken B, Sánchez CI. A survey on deep learning in medical image analysis. Medical image analysis. $2017 \mathrm{Dec}$ 1;42:60-88.

[5] Havaei M, Davy A, Warde-Farley D, Biard A, Courville A, Bengio Y, $\mathrm{Pal}$ C, Jodoin PM, Larochelle H. Brain tumor segmentation with deep neural networks. Medical image analysis. 2017 Jan 1;35:18-31.

[6] Rangarajan, P. "Brain tumour detection using discrete wavelet transform based medical image fusion." Biomedical Research28, no. 2 (2017)

[7] Qiu, Qingtao, Jinghao Duan, Guanzhong Gong, Yukun Lu, Dengwang $\mathrm{Li}$, Jie Lu, and Yong Yin. "Reproducibility of radiomic features with GrowCut and GraphCut semiautomatic tumor segmentation in hepatocellular carcinoma." Translational Cancer Research 6, no. 5 (2017): 940-948.

[8] Damodharan, Selvaraj, and Dhanasekaran Raghavan. "Combining Tissue Segmentation and Neural Network for Brain Tumor Detection." International Arab Journal of Information Technology (IAJIT) 12, no. 1 (2015)

[9] Alfonse, Marco, and Abdel-Badeeh M. Salem. "An automatic classification of brain tumors through MRI using support vector machine." Egyptian Computer Science Journal (2016)

[10] Ain, Quratul, M. Arfan Jaffar, and Tae-Sun Choi. "Fuzzy anisotropic diffusion based segmentation and texture based ensemble classification of brain tumor." Applied soft computing21 (2014): 330340.

[11] Abdel-Maksoud, E., Elmogy, M. and Al-Awadi, R., 2015. Brain tumor segmentation based on a hybrid clustering technique. Egyptian Informatics Journal, 16(1), pp.71-81.

[12] Zanaty, E. A. "Determination of gray matter (GM) and white matter (WM) volume in brain magnetic resonance images (MRI)." International Journal of Computer Applications 45, no. 3 (2012).

[13] Kong, Y., Deng, Y., \& Dai, Q. (2015). Discriminative clustering and feature selection for brain MRI segmentation. IEEE Signal Processing Letters, 22(5), 573-577.

[14] Demirhan, Ayşe, Mustafa Törü, and İnan Güler. "Segmentation of tumor and edema along with healthy tissues of brain using wavelets and neural networks." IEEE journal of biomedical and health informatics 19, no. 4 (2015): 1451-1458

[15] Torheim, Turid, Eirik Malinen, Knut Kvaal, Heidi Lyng, Ulf G. Indahl, Erlend KF Andersen, and Cecilia M. Futsaether "Classification of dynamic contrast enhanced MR images of cervical cancers using texture analysis and support vector machines." IEEE transactions on medical imaging 33, no. 8 (2014): 1648-1656.

[16] Guo, Lei, Lei Zhao, Youxi Wu, Ying Li, Guizhi Xu, and Qingxin Yan. "Tumor detection in MR images using one-class immune feature weighted SVMs." IEEE Transactions on Magnetics 47, no. 10 (2011): 3849-3852.

[17] Kumar, P., and B. Vijayakumar. "Brain tumour $\mathrm{Mr}$ image segmentation and classification using by PCA and RBF kernel based support vector machine." Middle-East Journal of Scientific Research 23, no. 9 (2015): 2106-2116.

[18] Sharma, Neeraj, Amit K. Ray, Shiru Sharma, K. K. Shukla, Satyajit Pradhan, and Lalit M. Aggarwal. "Segmentation and classification of medical images using texture-primitive features: Application of BAMtype artificial neural network." Journal of medical physics/Association of Medical Physicists of India 33, no. 3 (2008): 119

[19] Cui, Wenchao, Yi Wang, Yangyu Fan, Yan Feng, and Tao Lei. "Localized FCM clustering with spatial information for medical image segmentation and bias field estimation. "Journal of Biomedical Imaging 2013 (2013): 13.

[20] Wang, Guodong, Jie Xu, Qian Dong, and Zhenkuan Pan. "Active contour model coupling with higher order diffusion for medical image segmentation." International journal of biomedical imaging 2014 (2014).

[21] Deepa, S. N., and B. Arunadevi. "extreme learning machine for classification of brain tumor in $3 \mathrm{~d} \mathrm{mr}$ images/elm za klasifikaciju tumora mozga kod 3d mr snimaka." Informatologia 46, no. 2 (2013): 111.

[22] Olabarriaga S, Smeulders A. Interaction in the segmentation of medical images: a survey. Med Image Anal 2001, 5. pp 127-42.
[23] Foo JL. A survey of user interaction and automation in medical image segmentation methods. Tech rep ISUHCI20062, Human Computer Interaction Department, Iowa State Univ; 2006

[24] Bezdek JC, Hall LO, Clarke LP. Review of MR image segmentation techniques using patternrecognition. Med Phys. 1993 , (20) , pp 1033 48

[25] Liu, Jin, Min Li, Jianxin Wang, Fangxiang Wu, Tianming Liu, and Y Pan. "A survey of MRI-based brain tumor segmentation methods." Tsinghua Science and Technology 19, no. 6 (2014): 578595.

[26] Singh, Amritpal. "Detection of brain tumor in MRI images, using combination of fuzzy c-means and SVM." In Signal Processing and Integrated Networks (SPIN), 2015 2nd International Conference on, pp. 98-102. IEEE, 2015

[27] Yao, Jianhua, Jeremy Chen, and Catherine Chow. "Breast tumor analysis in dynamic contrast enhanced MRI using texture features and wavelet transform." IEEE Journal of selected topics in signal processing 3, no. 1 (2009): 94-100.

[28] Nanthagopal, A. Padma, and R. Sukanesh. "Wavelet statistical texture features-based segmentation and classification of brain computed tomography images." IET image processing 7, no. 1 (2013): 25-32

[29] Filippi M1, Horsfield MA, Bressi S, Martinelli V, Baratti C, Reganat $\mathrm{P}$, Campi A, Miller DH, Comi G. Intra- and inter-observer agreement of brain MRI lesion volume measurements in multiple sclerosis. A comparison of techniques. Brain 118(6), 1593-1600 (1995). 